\title{
Xanthophyll-cycle dependence of the energy transfer between carotenoid dark states and chlorophylls in NPQ mutants of living plants and in LHC II
}

\author{
Stefan Bode ${ }^{\mathrm{a}, 1}$, Claudia C. Quentmeier ${ }^{\mathrm{a}, 1}$, Pen-Nan Liao ${ }^{\mathrm{a}}$, \\ Tiago Barros ${ }^{c}$, Peter J. Walla ${ }^{\mathrm{a}, \mathrm{b}, *}$ \\ ${ }^{a}$ University of Braunschweig, Institute for Physical and Theoretical Chemistry, Department for Biophysical Chemistry, \\ Hans-Sommer-Strasse 10, 38106 Braunschweig, Germany \\ ${ }^{\mathrm{b}}$ Max-Planck-Institute for Biophysical Chemistry, Department of Spectroscopy and Photochemical Kinetics, Am Faßberg 11, 37077 Göttingen, Germany \\ ${ }^{c}$ Max-Planck-Institute of Biophysics, Department of Structural Biology, Max-von-Laue-Strasse 3, D-60438 Frankfurt am Main, Germany
}

Received 21 September 2007; in final form 6 November 2007

Available online 19 November 2007

\begin{abstract}

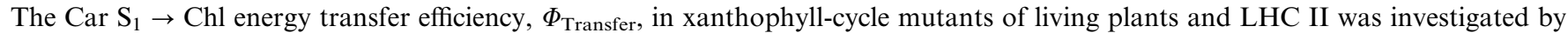
selective Car $\mathrm{S}_{1}$ two-photon excitation. Before high-light illumination $\Phi_{\text {Transfer }}$, of the violaxanthin deficient mutant $n p q 2$ is $\sim 30 \%$ smaller than the corresponding value for wild type plants. For the zeaxanthin deficient mutant, $n p q 1, \Phi_{\text {Transfer }}$ is $\sim 30 \%$ larger. Wild type Arabidopsis thaliana is the only variant which is capable of a light-dependent decrease of up to $40 \%$ and complete recovery to the original $\Phi_{\text {Transfer values. In contrast, }} \Phi_{\text {Transfer }}$ remains constant during dark adaptation in both mutants. Surprisingly, changes in $\Phi_{\text {Transfer }}$ of LHC II preparations were less than $5 \%$ only, when substituting violaxanthin by zeaxanthin.
\end{abstract}

(C) 2007 Elsevier B.V. All rights reserved.

\section{Introduction}

Light is necessary for the photosynthetic function and growth of plants. However, because light intensities vary over several orders of magnitude during a typical day, plants need to be able to quickly regulate their photosynthetic activity as a response to these changes [1]. Under low light conditions a maximum utilization of the energy is required for growth and survival. Under saturating high-light conditions the photosynthetic apparatus has to be protected from photo oxidative damage caused by excess energy. Plants regulate the photosynthetic activity

\footnotetext{
${ }^{*}$ Corresponding author. Address: University of Braunschweig, Institute for Physical and Theoretical Chemistry, Department for Biophysical Chemistry, Hans-Sommer-Strasse 10, 38106 Braunschweig, Germany. Fax: +495313915352.

E-mail address: pwalla@gwdg.de (P.J. Walla).

${ }^{1}$ These authors contributed equally to this work.
}

by quickly changing the balance between energy dissipation and energy utilization. Several attempts have been performed in order to understand the functioning of these mechanisms. But even though numerous explanations for the underlying mechanisms have been proposed no clear answer could be found so far [2-5].

One important change in the photosynthetic apparatus under high-light conditions is the transformation of the xanthophyll-cycle carotenoid violaxanthin (Vio) to zeaxanthin (Zea) by the enzyme violaxanthin de-epoxidase [6]. The importance of zeaxanthin in quenching processes has been demonstrated in many experiments but its exact role still remains unclear.

One of the quenching models proposed is the molecular gear shift mechanism which includes the optically dark $S_{1}$ states, Car $\mathrm{S}_{1}$, of the xanthophylls-cycle carotenoids Vio and Zea (see Fig. 1). The conjugated $\pi$ electron system of Zea has 11 double bonds whereas Vio has only nine. With a more extended conjugated $\pi$ electron system resulting in a 


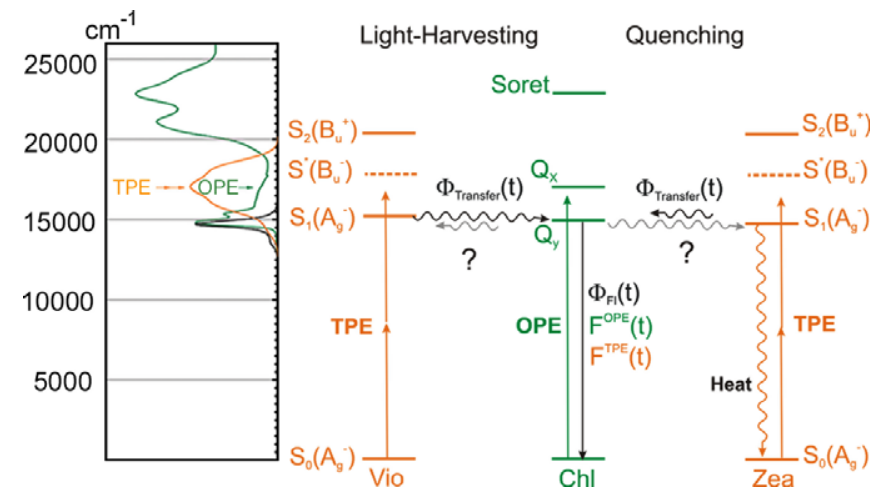

Fig. 1. Left - Green line: one-photon absorption of LHC II. Black line: fluorescence of LHC II after excitation at $592 \mathrm{~nm}$. Red line: Car $\mathrm{S}_{1}$ twophoton absorption spectrum of $\beta$-carotene as an example for typical carotenoids in photosynthetic pigment-protein complexes [15]. Right energy diagram of LHC II, Vio: violaxanthin, Chl: chlorophyll, Zea: zeaxanthin. For details see text.

lower energy level, the energy of the Zea $S_{1}$ level possibly drops below the lowest chlorophyll state, Chl $\mathrm{Q}_{\mathrm{y}}$, while the Vio $S_{1}$ level is slightly higher. Thus, the Zea $S_{1}$ state might function as an energy trap for excess energy [7]. Instead of being transferred to the reaction center of the photosynthetic apparatus a fraction of the excess energy could be dissipated as heat via carotenoid dark states.

Two recent studies indicated that such or a similar mechanism could indeed contribute to the regulation of plant photosynthesis. In one study Berera et al. showed that even the change of one double bond in the length of a conjugated carotenoid $\pi$-system suffices to switch from Car $\mathrm{S}_{1} \rightarrow \mathrm{Chl}$ to $\mathrm{Chl} \rightarrow$ Car $\mathrm{S}_{1}$ energy transfer [8]. In another study, Wehling and Walla showed that, in living plants, the adaptation to high-light conditions is closely correlated with a substantial change in the overall Car $\mathrm{S}_{1} \rightarrow$ Chl energy transfer efficiency, $\Phi_{\text {Transfer }}[9]$.

Due to the optically forbidden character of the Car $\mathrm{S}_{1}$ state it is not easy to gain insight into energy transfer and quenching mechanisms in which this state might participate [10]. One possibility for direct investigation of the energy transfer involving the carotenoid dark states, Car $S_{1}$, is via two-photon excitation (TPE) because $A_{g}^{-} \rightarrow A_{g}^{-}$ transitions are generally two-photon allowed (Fig. 1) [1114]. TPE has proven a strong tool in the research of photosynthesis and photoprotection [15-17].

Usually, the extent of the excitation energy quenching in plants is observed by monitoring the residual fluorescence emitted by the chlorophyll $(\mathrm{Chl})$ pigments in the photosynthetic apparatus [18]. The chlorophyll fluorescence after conventional one-photon excitation $F^{\mathrm{OPE}}$ is directly proportional to the amount (quantum yield) of excitation energy that has not been quenched by reaction center photochemistry or any other quenching process

$F^{\mathrm{OPE}}(t)=I_{\mathrm{Abs}}^{\mathrm{OPE}} \cdot \Phi_{\mathrm{Fl}}(t)$

Here, $I_{\mathrm{Abs}}$, defines simply the light intensity or number of photons absorbed by the pigments. Of course, the chlo- rophyll fluorescence observed after selective TPE of the carotenoid dark states is additionally proportional to the quantum efficiency of the excitation energy transfer, $\Phi_{\text {Transfer }}$, from the carotenoid dark states to the chlorophylls Car $\mathrm{S}_{1} \rightarrow$ Chl Q

$F^{\mathrm{TPE}}(t)=I_{\mathrm{Abs}}^{\mathrm{TPE}} \cdot \Phi_{\mathrm{Fl}}(t) \cdot \Phi_{\mathrm{Transfer}}(t)$

Thus, a significant increase or decrease in the measured ratio

$\Phi_{\text {Transfer }}(t) \propto \frac{F^{\mathrm{TPE}}(t)}{F^{\mathrm{OPE}}(t)}$

during the adaptation processes indicates an increase or decrease in the carotenoid dark state to chlorophyll energy transfer. In [9], Wehling and Walla demonstrated that $\Phi_{\text {Transfer }}$ decreases significantly in most of the investigated plants during the adaptation to high-light conditions. Several questions arise: is this phenomenon indeed correlated with the plants xanthophyll cycle and changing carotenoid compositions or are other processes relevant? Does $\Phi_{\text {Transfer }}$ recover back to its original level during dark adaptation? Which pigment-protein complex or complexes and/or regulation mechanism in the photosynthetic apparatus is the origin of the observed drastic energy transfer changes? A straight forward approach to gain insights into these questions is to determine $\Phi_{\text {Transfer }}$ for xantophyll cycle mutants of plants and isolated pigment-protein complexes of the photosynthetic apparatus, which have varying levels in the xanthophyll composition corresponding to the levels found in plants under high- or low-light conditions.

In this context, two xanthophyll-cycle-mutants of $\mathrm{Ara}$ bidopsis thaliana are especially useful: In the mutant $n p q I$ the enzymatic conversion from Vio to Zea is blocked and thus it contains almost exclusively Vio. In contrast, in the mutant $n p q 2$ the enzymatic conversion from Zea to Vio is blocked and thus it contains almost exclusively Zea $[2,19,20]$. In the present study, we investigated light-dependent changes in $\Phi_{\text {Transfer }}$ of these mutants and compared them directly to $\Phi_{\text {Transfer }}$ observed for wild type plants. Additionally, we investigated preparations of the most abundant light-harvesting complex LHC II containing differing amounts in the xanthophyll cycle carotenoids to find out, if LHC II is the origin of the observed energy transfer changes and thus might be the decisive site for photosynthetic regulation.

\section{Materials and methods}

The laser system consisted of a Vitesse Duo providing the pump beam and the high repetition pulses by the integrated oscillator (see Fig. 2). The ultrashort pulses were amplified with a RegA 9000 and used for pumping an optical parametric amplificator (OPA 9450) (all components from Coherent Inc., Santa Clara, CA). The system was set to a repetition rate of $120 \mathrm{kHz}$. The OPA provided 


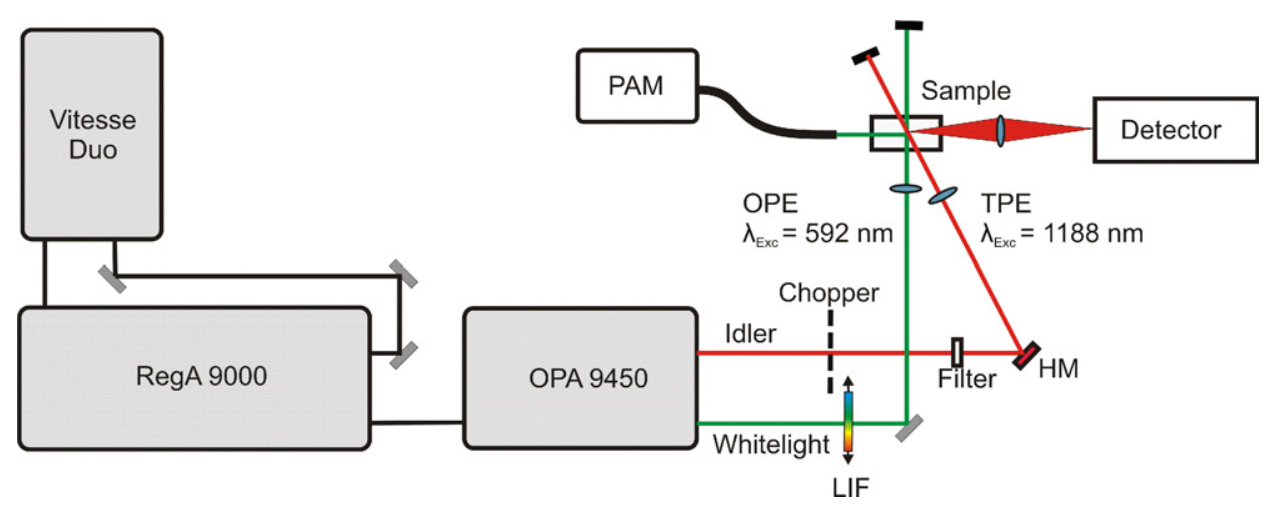

Fig. 2. Experimental set-up. For two-photon excitation the idler beam is used. For one-photon excitation the white light of the OPA or the PAM is used, respectively, HM: hot mirror. For details see text.

pulses of $90 \mathrm{fs}$ FWHM $\left(\lambda_{\text {Signal }} \sim 600 \mathrm{~nm}, \lambda_{\text {Idler }} \sim 1200 \mathrm{~nm}\right)$ and a broad white-light spectrum $(\lambda=400-2000 \mathrm{~nm})$. For the two-photon excitation of the carotenoids the idler beam was used $(1200 \mathrm{~nm}, \sim 12.5 \mathrm{~nJ}$ per pulse). A hot mirror (L46-386, Edmund Optics) and an additional IR filter (long pass, $900 \mathrm{~nm}$ cut-off, Edmund Optics) were used for a complete rejection of the visible light of the signal beam. A spectrally filtered fraction $(590 \pm 20 \mathrm{~nm}, \sim 0.075 \mathrm{~nJ}$ per pulse) of the white-light continuum of the OPA was used for one-photon excitation of the LHC II samples. A gradient interference filter was used to pick the desired wavelength for excitation. Both beams were focused into the sample with achromatic lenses $(f=5 \mathrm{~cm}$ and $f=10 \mathrm{~cm}$, respectively) mounted on three-dimensional translation stages. For one-photon excitation of the plants a commercial PAM system (Pulse Amplified Modulation, Heinz Walz GmbH, Effeltrich, Germany) was used [21]. The PAM is integrated into the fluorescence detection system with a confocal arrangement.

For fluorescence detection after two-photon excitation an ultrafast photodiode (design by Prof. D. Schwarzer) connected to a lock-in amplifier (EG\&G 5205, Dumberry,
Canada) was used. The lock-in amplifier was synchronized by a chopper positioned in the idler beam path.

With lock-in amplified detection of the two-photon fluorescence and a commercial PAM for the detection of the one-photon fluorescence it is possible to measure under standard conditions with respect to actinic light irradiation, using far red excitation, and to measure changes in the Car $\mathrm{S}_{1} \rightarrow \mathrm{Chl}$ quantum efficiency in plants. Because this detection scheme allows measuring both, $F^{\mathrm{OPE}}$ and $F^{\mathrm{TPE}}$, by saturating pulses, it allows for the first time to determine $\Phi_{\text {Transfer }}$ also during dark adaptation (Fig. 3).

For fluorescence lifetime measurements a Ti:Sa laser with a central wavelength of $\sim 800 \mathrm{~nm}$ was used for twophoton excitation of the Car $\mathrm{S}_{2}$ and the chlorophyll Soret bands around $400 \mathrm{~nm}$. The Ti:Sa provided pulses of $100 \mathrm{fs}$ at a repetition rate of $87 \mathrm{MHz}$. A fraction of the excitation light $(20-30 \mathrm{~mW})$ was sent into a water immersion objective (UApo, $40 \times / 1.15$ water immersion, Olympus). The fluorescence light passed a dichroic beamsplitter (DC $590 \mathrm{~nm}$ long pass, AHF Analysentechnik, Tübingen, Germany) and was focused with a $100 \mathrm{~mm}$ achromatic lens onto an avalanche photo diode (SPCM-AQR-13, Perkin-
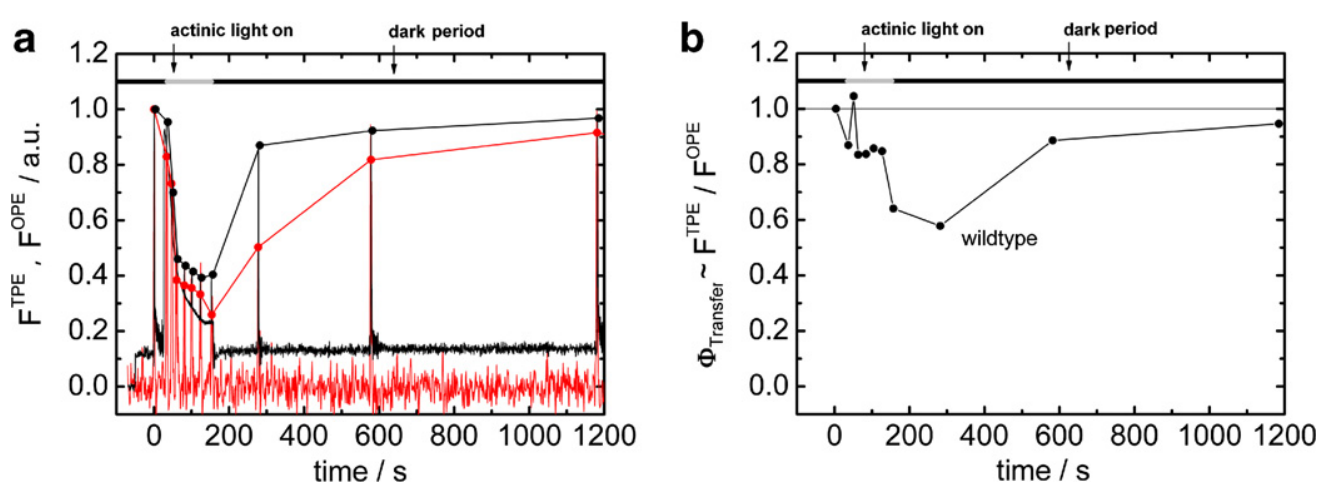

Fig. 3. Example of the determination of $\Phi_{\text {Transfer }}$ for a single Arabidopsis thaliana plant with the improved set-up. (a) Saturating OPE pulses from a standard PAM device were used to determine $F^{\mathrm{OPE}}(t)$ (black line with black circles, actinic light corresponded to $380 \mu \mathrm{mol} \mathrm{photons} / \mathrm{m}^{-2} \mathrm{~s}^{-1}$, saturating pulses corresponded to $\sim 4800 \mu \mathrm{mol}$ photons $/ \mathrm{m}^{-2} \mathrm{~s}^{-1}$ ). In addition $F^{\mathrm{TPE}}(t)$ was determined using saturating TPE pulses (red line with red circles; the TPE intensity corresponded to a similar amount of excitation quanta as OPE). (b) Plot of $\Phi_{\text {Transfer }}$ as calculated from $F^{\mathrm{TPE}}(t)$ and $F^{\mathrm{OPE}}(t)$ observed during saturating TPE and OPE pulses (Eq. (3)). After actinic high-light irradiance a decrease in the transfer coefficient is observed. After 15 min the original level is nearly reached again. 
Elmer, Dumberry, Kanada). Single-photon counting and fluorescence lifetime analysis were performed by using a timeharp 200 TCSPC (time correlated single-photon counting) card (Picoquant, Berlin). For details of the set-up see Pohl et al. [22]. Absorption spectra and fluorescence spectra of the LHC II preparations were recorded on a UV/ vis spectrometer from Perkin-Elmer Lambda and a Cary Eclipse fluorescence spectrometer from Varian, respectively.

A. thaliana plants of ecotype Columbia- 0 were grown in low nutrient soil at light intensities of $200 \mu \mathrm{mol}$ photons $\mathrm{m}^{-2} \mathrm{~s}^{-1}$ and a constant temperature of $21^{\circ} \mathrm{C}$ with an humidity of $70 \%$ under long day conditions ( $14 \mathrm{~h}$ light, $10 \mathrm{~h}$ dark). The wild type, $n p q 1$ and $n p q 2$ seeds were purchased at NASC (The European Arabidopsis Stock Info).

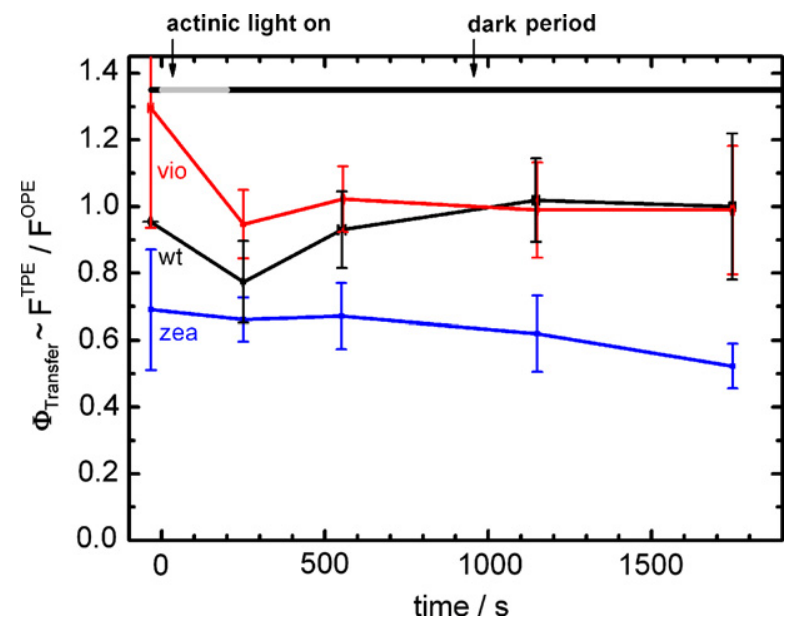

Fig. 4. Comparison of $\Phi_{\text {Transfer }}$ observed with Arabidopsis thaliana (black data) with $\Phi_{\text {Transfer }}$ observed with the mutants $n p q 1$ (red data) and $n p q 2$ (blue data) before ( $0 \mathrm{~s}$ ) and after high-light illumination (after $250 \mathrm{~s})$. To obtain mean values and standard deviations for each curve at least five different plants from different generations were measured. Actinic light was on during the light-grey period. The error bars for the initial $\Phi_{\text {Transfer }}$ values (before actinic light was on, time $<0 \mathrm{~s}$ ) relate to the standard deviations relative to the wild type value (Table 2) whereas error bars given for the dark period (time $>0 \mathrm{~s}$ ) relate to the variations in $\Phi_{\text {Transfer }}$ relative to the initial values of the same variant.

Table 1

Sample composition measured with HPLC [27]

\begin{tabular}{llllll}
\hline Molecule & Isolated & & & \multicolumn{2}{l}{ Reconstituted } \\
\cline { 2 - 3 } \cline { 5 - 6 } & $\begin{array}{l}\text { Vio } \\
\text { enriched }\end{array}$ & $\begin{array}{l}\text { Zea } \\
\text { enriched }\end{array}$ & & $\begin{array}{l}\text { Vio } \\
\text { enriched }\end{array}$ & $\begin{array}{l}\text { Zea } \\
\text { enriched }\end{array}$ \\
\hline Neoxanthin & 0.91 & 0.94 & 0.71 & 0.75 \\
Violaxanthin & 0.53 & 0.28 & 0.21 & 0.20 \\
Antheraxanthin & 0 & 0.07 & 0 & 0 \\
Lutein & 2.27 & 2.43 & & 2.21 & 1.94 \\
Zeaxanthin & 0 & 0.14 & 0 & 0.36 \\
Chl b & 6.04 & 6.08 & 5.37 & 5.71 \\
Chl a & 7.96 & 7.92 & 8.63 & 8.29 \\
\hline
\end{tabular}

Pigment composition calculated per 14 chlorophyll monomer according to Gilmore et al. [27].
For the averaged results presented in Fig. 4 several different generations were used.

Native LHC II proteins were isolated from spinach as described previously $[23,24,30]$. For the preparation of reconstituted LHC II samples methods as described in $[25,26]$ were used. The monomer composition was determined by HPLC (Table 1). For the measurements the samples were diluted with an aqueous buffer of $0.05 \%$ DDM and $50 \mathrm{mM}$ Tris at a $\mathrm{pH}$ of 7.5.

\section{Results}

\subsection{Car $S_{1} \rightarrow$ Chl energy transfer efficiency during the regulation of intact wild type plants}

To quantify the qualitative results observed in our prior publication [9] with the improved PAM/TPE-set-up we first measured $F^{\mathrm{TPE}}(t)$ and $F^{\mathrm{OPE}}(t)$ from wild type leaves of $A$. thaliana during the adaptation to changing light conditions. In most cases we again observed a larger decrease in $F^{\mathrm{TPE}}(t)$ in comparison to $F^{\mathrm{OPE}}(t)$ after high-light adaptation (high light switched on between 30 and $150 \mathrm{~s}$ in Fig. 3). Please note that in the present work both, $F^{\mathrm{TPE}}(t)$ and $F^{\mathrm{OPE}}(t)$, are measured using saturating pulses. In Fig. 3 an example of a single plant of $A$. thaliana with an especially large decrease in $\Phi_{\operatorname{Transfer}}(t) \propto \frac{F^{\mathrm{TPE}}(t)}{F^{\mathrm{OPE}}(t)}$ of up to $40 \%$ is shown. During the dark period, the Car $\mathrm{S}_{1} \rightarrow \mathrm{Chl}$ energy transfer quantum efficiency recovers indeed back to the original level, which further stresses the strong correlation of $\Phi_{\text {Transfer }}$ with the regulation of plant photosynthesis. However, recovery to the original $\Phi_{\text {Transfer-level apparently lags }}$ somewhat behind the changing light conditions. This might be a result of a slower enzymatic interconversion of the xanthophyll cycle pigments and will be subject of future studies.

\subsection{Car $S_{1} \rightarrow$ Chl energy transfer during the regulation of xantophyll cycle mutants}

In a next step several leaves from different generations of the xantophyll cycle mutants $n p q 1$ and $n p q 2$ of $A$. thaliana were measured and compared to the absolute fluorescence intensities observed with wild type plants. Fig. 4 shows the corresponding average values and standard deviations of $\Phi_{\text {Transfer }}$ at different times before and after high-light illumination. The mean initial $\Phi_{\text {Transfer (before actinic light on in }}$ Fig. 4) in the zea enriched mutant (npq2) is by a factor of $0.69 \pm 0.18$ lower than the corresponding value for wild type plants whereas the mean initial $\Phi_{\text {Transfer }}$ in the $n p q 1$ mutant (vio enriched) is by a factor of $1.3 \pm 0.36$ higher. The $\Phi_{\text {Transfer }}$ of wild type A. thaliana was normalized to 1 (Table 2).

The only variant which shows a light-dependent decrease and recovery of the original $\Phi_{\text {Transfer }}$ values is wild type $A$. thaliana. The transfer efficiency of $n p q 2$ does not change after high-light illumination and remains more or less constant during the dark period. For $n p q 1$ the transfer 
Table 2

OPE and TPE fluorescence intensities and standard deviation normalized for wt to 1 and $\Phi_{\text {Transfer }}$ values for wt, $n p q 1$ and $n p q 2$ observed before actinic high-light illumination

\begin{tabular}{llll}
\hline & $F^{\mathrm{TPE}}$ & $F^{\mathrm{OPE}}$ & $\Phi_{\text {Transfer }}=F^{\mathrm{TPE}} / F^{\mathrm{OPE}}$ \\
\hline wt & 1 & 1 & 1 \\
$n p q 2$ & $0.62 \pm 0.18$ & $0.89 \pm 0.08$ & $0.69 \pm 0.18$ \\
$n p q 1$ & $1.37 \pm 0.36$ & $1.06 \pm 0.18$ & $1.3 \pm 0.36$ \\
\hline
\end{tabular}

efficiency also stays constant during the dark period, but has decreased significantly after the high-light period. This might be due to irreversible photo damaging processes in this variant, which does not contain any zeaxanthin and, consequently, is capable of only very little photoprotection.

\subsection{Car $S_{1} \rightarrow$ Chl energy transfer efficiency and Chl $\rightarrow$ Car $S_{1}$ energy transfer in LHC II preparations}

To elucidate whether the major pigment complex LHC II contributes significantly to the observed effects we investigated reconstituted and isolated LHC II samples containing differing xanthophyll cycle compositions with the same set-up.

The values of the transfer parameter $\Phi_{\text {Transfer }}$ obtained by four repetitions of the measurement are displayed in Fig. 5. The averaged values are listed in Table 3.

For a better comparison of all data, the mean $\Phi_{\text {Transfer }}$ values of violaxanthin enriched LHC II were normalized on 1 (black columns) and the corresponding mean $\Phi_{\text {Transfer }}$ values of zeaxanthin (red columns) were corrected accordingly. The data show that the transfer parameter, $\Phi_{\text {Transfer }}$, for the samples enriched with Zea is only slightly but significantly lower than the parameter obtained for the samples enriched with Vio. The results for the reconstituted samples are very similar and are displayed in the central part of Fig. 5. Such small differences can already be explained by

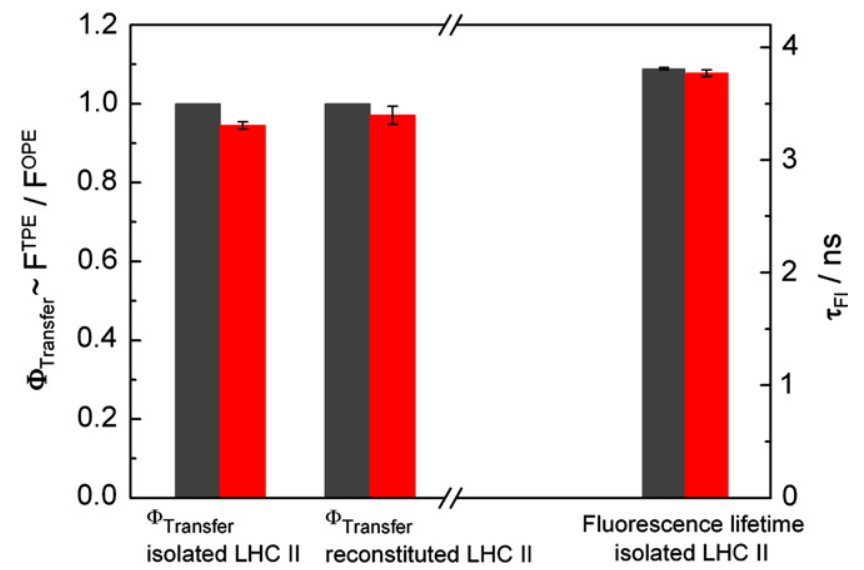

Fig. 5. Relative Car $\mathrm{S}_{1} \rightarrow$ Chl energy transfer efficiencies, $\Phi_{\text {Transfer, }}$, for the different types of LHC II preparations. Dark grey: samples containing high amounts of Vio, Red: samples with enriched amounts of Zea, Left: isolated LHC II with low amounts of Zea, Central: reconstituted LHC II samples, and Right: fluorescence lifetime of isolated LHC II samples.
Table 3

Relative Car $\mathrm{S}_{1} \rightarrow$ Chl energy transfer efficiencies, $\Phi_{\text {Transfer, of various }}$ LHC II preparations (from Fig. 5) and fluorescence lifetimes for the isolated samples, obtained by fitting monoexponential decay functions to the original data

\begin{tabular}{llll}
\hline Sample & Enriched & $\theta_{\text {Transfer }}$ & $\mathrm{sd}$ \\
\hline Isolated LHC II & Violaxanthin & 1 & - \\
& Zeaxanthin & 0.945 & 0.010 \\
Reconstituted LHC II & Violaxanthin & 1 & - \\
& Zeaxanthin & 0.971 & 0.023 \\
& & $\tau / \mathrm{ns}$ & $\mathrm{sd}$ \\
\cline { 2 - 4 } Isolated LHC II & Violaxanthin & 3.81 & 0.01 \\
& Zeaxanthin & 3.77 & 0.03 \\
\hline
\end{tabular}

potentially differing two-photon cross-sections of the carotenoids zeaxanthin and violaxanthin at the chosen TPE wavelength. However, this demonstrates the accuracy of using two-photon excitation for determination and comparison of Car $\mathrm{S}_{1} \rightarrow \mathrm{Chl}$ transfer efficiencies even in cases where the carotenoids are exchanged and that the significant changes in $\Phi_{\text {Transfer }}$ observed for plants cannot be explained simply by different two-photon absorption cross-sections of the carotenoids.

To investigate additionally a potential influence of the xanthophyll cycle composition on $\mathrm{Chl} \rightarrow \mathrm{Car} \mathrm{S}_{1}$ energy transfer we characterized the isolated LHC II by its fluorescence lifetime (right part Fig. 5). A decrease or increase in fluorescence lifetime would directly indicate a decrease or increase in $\mathrm{Chl} \rightarrow \mathrm{Car} \mathrm{S}_{1}$ energy transfer efficiency, respectively.

For a most comparable description of the fluorescence lifetime a decay curves using only a mono-exponential fitting function was done. Both native samples, enriched with Zea or Vio, respectively, have a lifetime of around $3.8 \mathrm{~ns}$. Within the standard deviations no significant difference in the fluorescence lifetimes can be observed (Table 3). These results are in good agreement with results obtained by Bassi et al. [28].

\section{Discussion and conclusions}

The improved two-photon set-up used for the present investigation allowed for the first time to quantify the recently observed decrease of the overall Car $\mathrm{S}_{1} \rightarrow \mathrm{Chl}$ energy transfer, $\Phi_{\text {Transfer }}$, in wild type plants [9]. Absolute values in the relative decrease in $\Phi_{\text {Transfer }}$ of up to $\sim 40 \%$ during high-light adaptation of wild type plants under saturating pulse conditions could be determined. In addition, it allowed for the first time to observe a complete recovery of $\Phi_{\text {Transfer }}$ back to the original level during dark adaptation of the plants. The observed small time-lag in the recovery in $\Phi_{\text {Transfer }}$ after the high-light period is probably an important detail for understanding the question, why the regulation happens faster than the actual enzymatic xanthophyll interconversion. All measurements were done under standard conditions of a commercial PAM fluorim- 
eter (including far-red excitation). It was also shown for the first time that before high-light conditions the violaxanthin-deficient mutant $n p q 2$ has a $~ 30 \%$ lower transfer efficiency whereas the zeaxanthin-deficient mutant npql has a $\sim 30 \%$ higher transfer efficiency compared to wild type plants. Wild type $A$. thaliana is the only variant which is capable of a light-dependent decrease and recovery to the original $\Phi_{\text {Transfer }}$ values. For both mutants lacking an intact xanthophyll cycle also the transfer efficiency stays constant during dark period. It is puzzling, however, that the transfer efficiency of $n p q 1$ declines significantly after high-light adaptation. This might be due to irreversible processes in the variant which does not contain any photoprotective zeaxanthin.

For example, it has been shown for $n p q 1$ and $n p q 2$ of Chlamydomonas rheinhardtii that only the zeaxanthin enriched mutant $n p q 2$ is protected against high-light induced photosystem II inactivation resulting in a slowed-down D1 protein turnover [29]. Further experiments will be necessary to clarify the reasons for the observed decrease in $\Phi_{\text {Transfer }}$ in $n p q 1$ which is absent in $n p q 2$.

Nevertheless, the present results demonstrate clearly that a full correlation in the Car $\mathrm{S}_{1} \rightarrow \mathrm{Chl}$ energy transfer with the regulation of plant photosynthesis during highlight and low-light-adaptation requires the presence of an intact xanthophyll-cycle in the plants.

Looking at the most abundant light-harvesting complex, LHC II, we observed that differing contents of the xanthophyll cycle carotenoids, zeaxanthin and violaxanthin, in the major light-harvesting complex LHC II affects only very little the Car $\mathrm{S}_{1} \leftrightarrow \mathrm{Chl}$ energy transfer in isolated as well as in reconstituted samples. In the presence of zeaxanthin, the Car $\mathrm{S}_{1} \rightarrow$ Chl energy transfer efficiency decreases at most by $\sim 5.5 \pm 0.1 \%$ and $\sim 3 \pm 0.2 \%$, respectively. Also, the $\mathrm{Chl} \rightarrow \mathrm{Car} \mathrm{S}_{1}$ energy transfer efficiency does not change significantly, as measured by comparing the chlorophyll fluorescence lifetimes of the LHC II samples. This is surprising, given the large xanthophyll-dependent effects observed with plant mutants. A recent study by Amarie et al. [30] demonstrated that also no significant difference in the carotenoid radical cation concentration can be found in corresponding samples of LHC II. However, given the lack of a clear photo-physical model for the regulation processes it was no prerequisite that from the absence of xanthophyll-dependent changes in the carotenoid radical ion concentration also an absence in xanthophyll-dependent $\mathrm{Car} \mathrm{S}_{1} \rightarrow \mathrm{Chl}$ energy transfer changes can be concluded.

In sum, the present data constitute an important piece of information in the puzzle of the molecular mechanism of the regulation processes. The comparative determination of $\Phi_{\text {Transfer }}$ of wild type of $A$. thaliana and corresponding xanthophyll-cycle mutants under changing light-conditions provides evidence for the close correlation of the Car $S_{1} \rightarrow$ Chl energy transfer efficiency with the presence of an intact xanthophyll cycle, the xanthophyll composition as well as with the regulation of plant photosynthesis.
However, the data observed with preparations of LHC II containing different amounts of xantophylls demonstrated that LHC II is apparently not the main site responsible for the observed correlation. We thus plan to investigate other pigment-protein complexes of the photosynthetic apparatus in future studies in order to identify potential sites of zeaxanthin-dependent quenching. Also, aggregation-dependent investigations of the Car $\mathrm{S}_{1} \rightarrow \mathrm{Chl}$ energy transfer of LHC II-samples containing differing amounts of xanthyophyll cycle carotenoids will be the subject of future studies.

\section{Acknowledgements}

We thank W. Kühlbrandt for his kind collaboration, H. Betz for the excellent technical assistance with the LHC II preparations, Dr. F. Bittner for growing the Arabidopsis thaliana plants and I. Dreger for her help in preparing the manuscript. This work was financially supported by the Fonds der Chemischen Industrie, the Deutsche Forschungsgemeinschaft (DFG), the state of Lower Saxony, and the Federal Ministry of Education and Research (BMBF). TB is supported by the fellowship SFRH/BD/21440/2005 from Fundação para a Ciência e a Tecnologia.

\section{References}

[1] B. Demmig-Adams, W.W. Adams, Science 298 (2002) 2149.

[2] P. Horton, A.V. Ruban, R.G. Walters, Ann. Rev. Plant Physiol. Plant Mol. Biol. 47 (1996) 655

[3] P. Müller, X.-P. Li, K.K. Niyogi, Plant Physiol. 125 (2001) 1558.

[4] K.K. Niyogi, X.-P. Li, V. Rosenberg, H.-S. Jung, J. Exp. Botany 56 (2005) 375.

[5] N.E. Holt, D. Zigmantas, L. Valkunas, X.-P. Li, K.K. Niyogi, G.R. Fleming, Science 307 (2005) 433.

[6] B. Demmig-Adams, W.W. Adams, Trends Plant Sci. 1 (1996) 21

[7] H.A. Frank, A. Cua, V. Chynwat, A. Young, D. Gosztola, M.R. Wasielewski, Photosynth. Res. 41 (1994) 389.

[8] R. Berera et al., PNAS 103 (2006) 5343.

[9] A. Wehling, P.J. Walla, Photosynth. Res. 90 (2006) 101.

[10] P. Tavan, K. Schulten, Phys. Rev. B 36 (1987) 4337.

[11] P.J. Walla, P.A. Linden, C.P. Hsu, G.D. Scholes, G.R. Fleming, PNAS 97 (2000) 10808.

[12] P.J. Walla, J. Yom, B.P. Krueger, G.R. Fleming, J. Phys. Chem. B 104 (2000) 4799.

[13] A.P. Shreve, J.K. Trautman, T.G. Owens, A.C. Albrecht, Chem Phys. Lett. 170 (1990) 51

[14] S. Shima et al., J. Phys. Chem. A 107 (2003) 8052.

[15] M. Hilbert, A. Wehling, E. Schlodder, P.J. Walla, J. Phys. Chem. B 108 (2004) 13022.

[16] P.J. Walla, P.A. Linden, K. Ohta, G.R. Fleming, J. Phys. Chem. A 106 (2002) 1909.

[17] A. Wehling, P.J. Walla, J. Phys Chem. B 109 (2005) 24510.

[18] U. Schreiber, Photosynth. Res. 4 (1983) 361.

[19] K.K. Niyogi, O. Björkman, A.R. Grossman, Plant Cell 9 (1997) 1369.

[20] K.K. Niyogi, A.R. Grossman, O. Björkman, Plant Cell 10 (1998) 1121.

[21] U. Schreiber, Photosynth. Res. 9 (1986) 261.

[22] W.H. Pohl, H. Hellmuth, M. Hilbert, J. Seibel, P.J. Walla, Chem. Biol. Chem. 7 (2006) 268. 
[23] J.J. Burke, C.L. Ditto, C.J. Arntzen, Arch. Biochem. Biophys. 187 (1978) 252.

[24] W. Kühlbrandt, T. Thaler, E. Wehrli, J. Cell. Biol. 96 (1983) 1414.

[25] H. Rogl, K. Kosemund, I. Collinson, FEBS Lett. 432 (1998) 21.

[26] J. Standfuss, W. Kühlbrandt, J. Biol. Chem. 279 (2004) 36884.

[27] A.M. Gilmore, H.Y. Yamamoto, J. Chromatogr. 543 (1991) 137.
[28] I. Moya, M. Silvestri, O. Vallon, G. Cinque, R. Bassi, Biochemistry 40 (2001) 12552.

[29] P. Jahns, D. Depka, A. Trebst, Plant Physiol. Biochem. 38 (2000) 371.

[30] S. Amarie, J. Standfuss, T. Barros, W. Kühlbrandt, A. Dreuw, J. Wachtveitl, J. Phys. Chem. B 111 (2007) 3481. 